\title{
Food Cue Reactivity, Obesity, and Impulsivity: Are They Associated?
}

\author{
Karolien van den Akker • Karen Stewart • Evangelia E. Antoniou • \\ Allison Palmberg • Anita Jansen
}

Published online: 20 September 2014

(C) Springer International Publishing AG 2014

\begin{abstract}
Heightened reactivity to food-associated cues and impulsive responding to these cues may be important contributors to the obesity epidemic. This article reviews the evidence for a role of food cue reactivity and impulsivity in food intake, body mass index, and weight-loss success. Inconsistencies in defining and measuring these constructs create difficulties in interpreting findings; however, evidence does support their role in obesity. The relationship between food cue reactivity and impulsivity may depend on the measurement used, but some studies have demonstrated that interactions between these constructs rather than direct effects are important in accounting for food intake pattern. Thus, multimodal assessment of both constructs is recommended. Future research would benefit from standardized definitions, measures, procedures, and reporting to enhance comparisons across studies. Implications for therapy are discussed and suggestions for further research are provided.
\end{abstract}

Keywords Food cue $\cdot$ Cue reactivity $\cdot$ Cue exposure $\cdot$ Eating behavior · Obesity $\cdot$ Impulsivity

Karolien van den Akker and Karen Stewart equally contributed to the writing of this article and are both considered the first author.

K. van den Akker $(\bowtie) \cdot$ E. E. Antoniou $\cdot$ A. Jansen

Faculty of Psychology and Neuroscience, Department of Clinical

Psychological Science, Maastricht University, P.O. Box 616, 6200

MD Maastricht, The Netherlands

e-mail: karolien.vandenakker@maastrichtuniversity.nl

K. Stewart

Department of Psychiatry, Virginia Commonwealth University, PO

Box 980710, Richmond, VA 23298-0710, USA

A. Palmberg

Department of Psychology, Virginia Commonwealth University, PO

Box 980710, Richmond, VA 23298-0710, USA

\section{Introduction}

Obesity is a major health problem worldwide. It has been estimated that, in the USA, obesity prevalence will increase from $32-35 \%$ in 2010 to $45-52 \%$ in 2030 [1]. There is a consensus that we now live in an "obesogenic environment" that promotes a sedentary lifestyle and provides an abundance of cues to eat hyperpalatable hypercaloric foods $[2,3]$. The omnipresence of food-associated cues is thought to stimulate food cravings, food intake, and potential weight gain [4].

This article reviews two constructs that may contribute to difficulties in regulating eating behavior in the obesogenic environment: food cue reactivity (physiologic and psychological responses to cues associated with eating that motivate eating behavior) [5] and impulsivity (rash action without full consideration of consequences) [6]. These two constructs present distinct, yet potentially related challenges to selfregulatory behavior, and promoting greater understanding of their role in the obesity epidemic may help in developing novel approaches to prevention and treatment. This review discusses the relationship of food cue reactivity and impulsivity with food intake, body mass index (BMI), and response to weight-loss interventions. These topics are also of interest in disordered and restrained eating; however, these eating behaviors are beyond the scope of the current review.

\section{Food Cue Reactivity}

Food cue reactivity comprises a series of physiologic and psychological responses to stimuli associated with eating that prompt an individual's motivation to eat. Although reactivity to food cues is largely influenced by genetics [7], it also has an important learned component. According to the cue reactivity model of overeating, changes in physiologic reactivity accompany changes in psychological reactivity, both learned 
through repeated pairings of food cues and subsequent food intake [5, 8]. Through Pavlovian conditioning, palatable food, which is a natural reinforcer (unconditioned stimulus), might become associated with both internal and external stimuli (conditioned stimuli). Such cues could include smells, images, locations, social situations, times of day, mood states, and food-related cognitions. As a result of these associations, conditioned stimuli come to elicit activation of a central appetitive state, resulting in a variety of physiologic and psychological responses [5, 8-12]. It is assumed that individuals who are highly susceptible to reward acquire associative learning for appetitive cues more readily [13]. Reactions to food cues that reflect this central appetitive state include psychological responses (e.g., craving, urge, or desire to eat), physiologic preparatory responses (e.g., the secretion of insulin and salivation) [5], and neurocognitive responses (e.g. brain activation patterns, allocation of attentional resources) [14]. The physiologic preparatory responses (cephalic phase responses) enable the organism to better digest, absorb, and metabolize nutrients [15] and, collectively, these responses motivate the organism to obtain and consume food.

Food cue reactivity is commonly measured using selfreport or direct observation methods. Self-report measures, such as the Power of Food (POF) scale [16] and the Reward Based Eating Drive (RED) scale [17] intend to measure multiple domains of psychological responding, including : cognitive ("It seems like most of my waking hours are preoccupied by thoughts about eating or not eating;" RED), affective ("If I see or smell a food I like, I get a powerful urge to have some;" POF), and behavioral ("When I start eating, I just can't seem to stop," RED) domains. Some studies use self-reported ratings of craving, desire to eat, or liking of food during food cue exposure $[18,19]$. Direct observation of behavioral responses to food cues typically involves monitoring of food intake during a bogus taste test in which participants are unaware that the amount they are consuming is being monitored [10]. Eye tracking and functional magnetic resonance imaging (fMRI) technologies can allow for measuring allocation of visual attention and observation of neurologic responses during exposure to food cues [e.g., 14, 20-25]. Physiologic reactivity to food cues is sometimes demonstrated using measures of salivation and gastric activity [26].

\section{Food Cue Reactivity, Food Intake, and BMI}

Findings of several studies suggest a positive relationship between food cue reactivity and BMI. Jansen et al. [22] reported cue-elicited salivation in overweight children that was highly predictive of the amount of calories consumed $(r=0.62)$. Ferriday and Brunstrom [27] also found that overweight participants reported a greater increase in the subjective desire to eat and increased salivation in response to the sight and smell of palatable food relative to normal-weight participants. Others found prolonged salivation to food cues, indicating less habituation to exposure to food cues, in obese individuals [28]. In addition, several prospective fMRI studies have shown that greater food cue-related activation in brain regions associated with reward and motivation predicts (a future increase in) BMI [23, 29-31].

Eye-tracking studies show some promise in documenting attentional biases, which might reflect a motivational state in response to food cues. In one study, participants were presented with food cues in two conditions: fasting and satiated. Results indicated no differences in gaze duration in the fasting condition. Yet, obese individuals in the "fed" condition displayed increased gaze duration on food cues compared with normal-weight individuals. This indicates that obese individuals remain attentive (motivated) to food cues in their environment regardless of actual satiety and hunger level [20]. Other studies have demonstrated attentional biases towards food in obese people [24, 32, 33]; however, some studies have found an opposite effect of BMI on attention to food targets using eye-tracking methods [22]. To explain these inconsistencies, it has been argued that attention bias is state dependent [34].

A systematic review of fMRI studies found that obese individuals show enhanced activation in reward centers when exposed to food cues compared with normal-weight individuals. This effect was particularly evident in response to high energy-dense foods and when exposure tasks were completed while participants were satiated [35], consistent with the observation that obese individuals maintain attention to food cues when satiated $[20,36]$.

Associations between physiologic and psychological measures of food cue reactivity are not strongly supported and further research is needed to clarify how reactivity relates to food intake patterns. In a study of normal-weight non-dieting women, participants reported subjective increases in craving and hunger during exposure to food cues and increases in heart rate, blood pressure, skin conductance, saliva production, and low-frequency heart rate variability were observed [37]. Cravings were correlated with changes in diastolic and systolic blood pressure, but not with any other physiologic measures. The only reactivity measure that was associated with actual subsequent food intake was an increase in subjective cravings during cue exposure [37].

Food intake was increased on a bogus taste test for overweight/obese women in a study that found that they also more frequently fixated their initial gaze on food images compared with normal-weight women. This bias was positively associated with subjective food cravings prior to the food exposure task [32]. Increasing BMI is also associated with more frequent subjective food cravings, which are associated with an increased intake of the specific foods craved [38] and eating in the absence of hunger because of increased 
cue reactivity [39]. Interestingly, in one food intake study, attentional bias for food cues, subjective hunger, and food intake were all correlated for normal-weight participants while food intake was not correlated with these two measures for overweight/obese participants [21]. Evidence appears to support a role of increased food cue reactivity in overweight/ obese individuals; however, the associations between the physiologic and psychological measures of reactivity are not well established. Although the link between different measures of food cue reactivity and subsequent eating behavior is still uncertain, there are indications that increased cue reactivity motivates eating, even in the absence of hunger and in excess of calories needed. Further research is needed to determine which measures of cue reactivity may best predict BMI and food intake.

\section{Food Cue Reactivity and Weight-Management Interventions}

It has been suggested that successful dieting is characterized by refraining from intake in response to specific food conditioned stimuli (food cues), resulting in extinction (i.e., inhibition) of the conditioned appetitive responses to these food cues [5]. Decreased salivation [40 ${ }^{\bullet}$ and increased inhibitory neural responses [41] to food cues have been noted in successful (formerly obese) weight losers compared with unsuccessful dieters and non-dieting obese controls. A prospective study has found that lower cue-elicited reward activation at baseline was predictive of increased weight loss during treatment [42•] and another study using a food-Stroop paradigm found that long-term successful dieters show significantly slower reaction times to food cues relative to both normalweight and obese individuals [43]. A systematic review of fMRI studies concluded that pre-post weight-loss comparisons show changes in activation patterns in brain areas involved in emotion, memory, and learning [35]. Another recent study showed that participating in a weight-loss intervention precipitated a shift in activation favoring low- over high-calorie foods [44]. Reductions in reward system activation in response to high-calorie foods accompanied by self-reported reductions in the desire to eat have also been noted in individuals 1 month after a Roux-en-Y gastric bypass [45].

Therefore, evidence supports the possibility that weightloss success is more readily attainable for individuals with relatively decreased sensitivity to food cues [12] and that both surgical and behavioral weight-loss interventions can promote changes in the reward system implicated in food cue reactivity. Further research is needed to reproduce and refine these results to provide better guidance in developing future obesity prevention and treatment programs.

\section{Impulsivity}

Impulsivity or a general tendency to act without sufficient consideration of consequences [6] has been conceptualized and measured in a number of ways over the years. Impulsivity consists of at least two facets: 1) rapid response impulsivity, i.e., problems with response inhibition that are typically measured using Go/No-Go, stop signal, continuous performance test, or similar cognitive tasks and 2) impulsive choice, i.e., difficulty forgoing immediate reward in favor of longer term benefit, which is often measured using delay discounting paradigms [46]. Impulsivity is also frequently measured using self-report measures such as the Barratt Impulsiveness Scale (BIS) [47] or the Urgency, (lack of) Premeditation, (lack of) Persistence, and Sensation-seeking scale (UPPS) [48]. Impulsivity can be both a trait and state characteristic [49].

\section{Impulsivity, Food Intake, and BMI}

Studies generally support a relationship between impulsivity and specific food intake patterns that would likely lead to weight gain. In a sample of overweight and obese women, those who were most impulsive on the delay discounting task chose the most calorie-dense food options when dining on ready-to-eat or take-away food. As a result, although they did not consume a greater volume of food, the impulsive individuals ingested more calories than the less impulsive participants [50]. Self-reported impulsivity is associated with unhealthy eating choices and eating for emotional reasons or external cues and poor Go/No-Go performance is associated with emotional eating and choosing less healthy food items [51]. Delay discounting, however, was not directly associated with food intake in another study of obese women [52•]. Following a state impulsivity manipulation, normal-weight non-dieting subjects consumed more food than those completing a control task [53•], showing that impulsivity might cause increased food intake. Some specific measures of impulsivity appear to be directly related to food intake; however, further multimodal assessment is necessary to further elucidate the aspects of impulsivity that most directly predict food intake.

Studies of impulsivity generally show that obesity is associated with less effective inhibitory control [54-62], greater self-reported impulsivity [63], and more impulsive choices $[64,65]$. Obese children show poor response inhibition using a stop-signal task compared with healthy-weight children [54, 55] and poor performance on this task is correlated with higher childhood BMI [55]. Obese adults report greater problems than normal-weight adults with urgency and lack of perseverance on the UPPS [63]. Obese women are more impulsive than their healthy-weight peers using delay discounting measures [57, 64], although some studies have failed to document 
differences in this measure related to BMI [58]. Obese women are also more impulsive on the stop-signal task [58], although some studies found that obese people only performed worse on the stop-signal task when food stimuli were used [56, 61]. It is noteworthy that a majority of obesity studies have focused on women, and that one study found increased (delay discounting) impulsivity in obese women, but not obese men [65], suggesting that further research on gender differences in impulsivity and food intake regulation is much needed.

Obesity may also contribute to impulsive behavior. Obesity is associated with deficits in cognitive performance, particularly executive function, a higher-level process that is involved in decision making and inhibitory control. Brain imaging studies in obese women confirm that difficult delay discounting tasks involve activation in regions that are implicated in executive function (middle and superior frontal gyri and inferior parietal lobule) and that more impulsive women showed less activation in these regions during the task [66]. The impact of obesity on cognition may be attributable to central effects of obesity-related systematic inflammation, insulin resistance, and lipid dysregulation, and the relationship between obesity and executive function is likely bi-directional [67]. Concurrent analyses show that poor executive function is related to high-calorie snack intake and sedentary behaviors in fourth graders [68] and accounts for discrepancies between intended and actual food intake in adults [69]. A few prospective studies are available to demonstrate that problems in executive function and impulsivity predate obesity and predict weight gain. Four-year-old children with difficulty delaying a reward had higher BMIs at a 30-year follow-up [70], and impulsive obese women (indicated by delay discounting and fMRI activation during this task) showed an accelerated rate of weight gain during follow-up (1.3-2.9 years) [71]. Additionally, the state impulsivity manipulation in Guierrieri et al. [53•] supports a causative role of impulsivity in overeating.

\section{Impulsivity and Weight-loss Interventions}

In the literature on substance use, trait impulsivity has been associated with severity of drug use problems and with poor treatment outcome [72, 73]. Similar evidence has been emerging in obesity treatment. Poor performance on a stop-signal task has been found to predict poorer weight-loss treatment outcome in obese children (mean age, 9.3 years) [55] and adolescents [50]. Another study did not find this to be the case in children, and found that poor performance on Go/No-Go and a complex interference task actually predicted better weight loss in adolescents [74]. An adult intervention study showed that delay discounting was associated with poor weight loss as was fMRI-measured activation and connectivity in prefrontal and anterior insula regions, which are implicated in impulse control and reward signaling [75]. These interventions differed significantly in their format (outpatient vs. inpatient), duration (range, 8-10 weeks to 1 year), focus (behavioral therapy only vs. multidisciplinary treatment vs. behavioral therapy with specific session content on impulse control vs. low calorie combined with nutritional counseling and physical activity), and patient populations (children vs. adolescents vs. adults). These discrepant findings highlight the need for additional treatment research with consideration of impulsivity and recognition that different approaches/intensity levels/duration of treatment may be needed for different individuals and different developmental stages.

\section{Impulsivity and Food Cue Reactivity}

Food cue reactivity and impulsivity commonly co-occur. In fact, individuals with both high cue reactivity and impulsive behavior may be at the greatest risk of overeating. In a sample of obese women, Appelhans and colleagues found that impulsivity moderated the relationship between food cue reactivity and food intake. Food cue reactivity was only associated with increased food intake in a bogus taste test for the participants who also performed poorly on the delay discounting task [53•]. Similar findings have been documented in normalweight women [76, see also 77]. With regard to the relationship between cue reactivity and impulsivity, some transdiagnostic studies demonstrate that they are correlated [78], while some do not [53•]. These discrepant findings are likely a result of variability in measurement, sample selection, and procedure.

It has been proposed that impulsivity may accelerate acquisition of Pavlovian conditioning to appetitive cues and some animal model evidence supports this [79], although human studies do not appear to bear out this theory. A recent study used augmented reality technology and Pavlovian conditioning to associate milkshake consumption with a specific virtual environment. Impulsive individuals did not differ from the non-impulsive individuals in acquisition of appetitive Pavlovian conditioning. However, following conditioning trials, impulsive individuals consumed more milkshake when exposed to the cue-associated augmented reality than in the non-cue-associated environment. This effect was not observed in non-impulsive individuals, suggesting that although they are not necessarily more likely to acquire conditioned cues to eat, impulsive individuals may be more susceptible to acting on acquired appetitive states once they have been established [80]. Other studies have failed to document evidence for differences in the acquisition of Pavlovian responses to food cues as a function of impulsivity [81]. 


\section{Clinical Implications}

The findings discussed have clinical implications: treatments that reduce impulsivity and food cue reactivity might be helpful in the reduction of overeating. One such treatment is cue exposure with response prevention, which aims to extinguish appetitive responses to previously reinforced cues. Although there is a need for large and well-controlled, randomized clinical trials, the available small-sized studies suggest that cue exposure with response prevention might be an effective intervention for lowering food cue reactivity and overeating in overweight and obese populations [5]. A neuroimaging study further showed that prolonged food cue exposure without eating leads to reduced reward-related activity in the brain [25].

A few studies have explicitly targeted impulsivity in weight-loss interventions. One novel approach by Veling and colleagues compared two Internet-based approaches: implementation intention vs. food Go/No-Go task. While both approaches improved weight loss compared with the control condition across the sample, implementation intention appeared to be especially effective for individuals with strong motivation. The Go/No-Go task enhanced weight loss in the individuals with the highest BMI whereas the implementation intervention was not efficacious in this group [82]. Another study shows that participants who learned to respond to healthy foods while inhibiting responses towards unhealthy foods, significantly reduced their snack intake in the weeks thereafter [83]. Comparable training effects were found for excessive drinking behavior: response inhibition training decreased alcohol consumption [84]. Working memory training in heavy drinkers was effective in decreasing alcohol intake [85]. In obese children, training of working memory was studied as part of a more general executive functioning training (the training of inhibition skills and working memory) [86]. The executive functioning training led to a significantly slower relapse (weight regain) at the 8-week follow-up, though this effect had disappeared at 12 weeks.

As discussed before, there is evidence that biased attention towards food predicts the strength of experienced cravings, the amount eaten, and even the amount of weight gained in obesity. It has been studied as to whether retraining of attention reduces the bias and related cravings. Studies demonstrate that training attention away from unhealthy foods favoring attention for healthy foods or neutral stimuli reduces food cravings and food intake [87-91].

Further research is needed to determine how best to address issues of food cue reactivity and impulsivity in overweight and obese individuals. Further advancements in the cognitive and behavioral interventions that may be well suited to these issues may emerge as we elucidate further the cognitive underpinnings of food cue reactivity and impulsivity.

\section{Conclusion}

Exposure to Pavlovian-conditioned food cues promotes psychological and physiologic appetitive states [37]. Overweight/ obese individuals appear to experience stronger responses to food cues [27] and consume more food than healthy-weight individuals in bogus taste tests [32]. Although some studies show that food cue reactivity is associated with greater food intake [22, 37-39], some evidence suggests that food cue reactivity only predicts food intake in highly impulsive individuals $[53 \cdot, 76]$. Being highly responsive to food cues is a negative prognostic factor for weight-loss success [42•], but weight loss also appears to reduce food cue reactivity [44].

Overweight/obese individuals appear to have more difficulty inhibiting responses [54], report more impulsive behaviors [63], and discount delayed rewards [65] more steeply than healthy-weight individuals. Impulsivity also appears to interfere with success in weight-loss interventions [92], although findings on this are mixed [74]. Obesity may thus be the result of acquired appetitive states in response to food cues coupled with diminished capacity for inhibiting behavioral response to these cues. Obesity in turn may maintain or even facilitate impulsive behavior as a result of central effects of systemic inflammation, insulin resistance, and lipid dysregulation that lead to reduced executive function [67].

The relationship between cue reactivity and impulsivity is complicated and likely depends on the method used to measure each construct and the population of interest. We recommend development of a standardized approach to defining and measuring food cue reactivity and impulsivity that will improve the comparison between studies with the overall effect of accelerating progress in obesity research. Studies have yet to clarify which physiologic and psychological measures of cue reactivity best predict eating behavior, BMI, and response to treatment, and impulsivity studies suffer from wide disparities in their approach to measuring impulsive behavior. Given the potential interaction between food cue reactivity and impulsivity, we recommend that future studies include multidimensional assessment of both food cue reactivity and impulsivity using measures and methodologies described in this article and in the referenced articles. Future studies should also focus on multi-method approaches to assessing eating behavior including the bogus taste test, food logs, and ecologic momentary assessment and should attend to multiple parameters of food intake (e.g., frequency of initiating an eating episode, volume of intake during an eating episode, calorie density of food intake). Randomized trials are needed to determine if specific intervention components work best for individuals with varying combinations and degrees of food cue reactivity and impulsivity. Finally, a majority of studies of obesity and weight-loss interventions have focused exclusively on women, and there is evidence to suggest that the relationships between impulsivity, food cue reactivity, and eating 
behavior may not be the same in men. Future studies should focus on men as well as women.

Acknowledgments This contribution is partially financed by the Netherlands Organisation for Scientific Research, Vici Grant 453.10.006, awarded to Anita Jansen.

iThenticate: $6 \%$

\section{Compliance with Ethics Guidelines}

Conflict of Interest Karolien van den Akker, Karen Stewart, Eva Antoniou, Allison Palmberg, and Anita Jansen declare no conflicts of interest.

Human and Animal Rights and Informed Consent This article does not contain any studies with human or animal subjects performed by the author.

\section{References}

Papers of particular interest, published recently, have been highlighted as:

\section{- Of importance}

1. Wang YC, McPherson K, Marsh T. Health and economic burden of the projected obesity trends in the USA and the UK (vol 378, pg 815, 2011). Lancet. 2011;378(9805):1778.

2. Swinburn B, Egger G, Raza F. Dissecting obesogenic environments: the development and application of a framework for identifying and prioritizing environmental interventions for obesity. Prev Med. 1999;29(6):563-70.

3. Goran MI, Weinsier RL. Role of environmental vs. metabolic factors in the etiology of obesity: time to focus on the environment. Obes Res. 2000;8(5):407-9.

4. Polivy J, Herman CP, Coelho JS. Caloric restriction in the presence of attractive food cues: external cues, eating, and weight. Physiol Behav. 2008;94(5):729-33.

5. Jansen A. A learning model of binge eating: cue reactivity and cue exposure. Behav Res Ther. 1998;36(3):257-72.

6. Moeller FG et al. Psychiatric aspects of impulsivity. Am J Psychiatr. 2001;158(11):1783-93

7. Carnell $\mathrm{S}$ et al. Genetic influence on appetite in children. Int J Obes. 2008;32(10):1468-73.

8. Jansen A, Havermans RC, Nederkoorn C. Cued overeating. In: Handbook of behavior, food and nutrition. Springer; 2011. p. 1431-1443.

9. Bouton ME. Learning and behavior: a contemporary synthesis. Sunderland, Massachusetts: Sinauer Associates; 2007.

10. Fedoroff IC, Polivy J, Herman CP. The effect of pre-exposure to food cues on the eating behavior of restrained and unrestrained eaters. Appetite. 1997;28(1):33-47.

11. Pavlov IP. Conditioned Reflexes. London: Oxford University Press; 1927.

12. Jansen A, Havermans R, Nederkoorn C. Cued overeating. In: Preedy VR, Watson RR, Martin CR, editors. Handbook of behavior, food and nutrition. New York: Springer; 2011. p. 1431-43.

13. Corr PJ, Pickering AD, Gray JA. Personality and reinforcement in associative and instrumental learning. Personal Individ Differ. 1995;19(1):47-71.

14. Yokum S, Ng J, Stice E. Attentional bias to food images associated with elevated weight and future weight gain: an fMRI study. Obesity. 2011;19(9):1775-83.
15. Power ML, Schulkin J. Anticipatory physiological regulation in feeding biology: cephalic phase responses. Appetite. 2008;50(2-3): 194-206.

16. Lowe MR et al. The power of food scale. A new measure of the psychological influence of the food environment. Appetite. 2009;53(1):114-8.

17. Epel ES et al. The reward-based eating drive scale: a self-report index of reward-based eating. PLoS One. 2014;9(6):e101350.

18. Vanstrien $\mathrm{T}$ et al. The predictive-validity of the dutch restrained eating scale. Int J Eat Disord. 1986;5(4):747-55.

19. Nijs IMT, Franken IHA, Muris P. The modified trait and state foodcravings questionnaires: development and validation of a general index of food craving. Appetite. 2007;49(1):38-46.

20. Castellanos EH et al. Obese adults have visual attention bias for food cue images: evidence for altered reward system function. Int $\mathrm{J}$ Obes. 2009;33(9):1063-73.

21. Nijs IM et al. Differences in attention to food and food intake between overweight/obese and normal-weight females under conditions of hunger and satiety. Appetite. 2010;54(2):243-54.

22. Nummenmaa $L$ et al. Food catches the eye but not for everyone: a BMI-contingent attentional bias in rapid detection of nutriments. PLoS One. 2011;6(5):e19215.

23. Stoeckel LE et al. Widespread reward-system activation in obese women in response to pictures of high-calorie foods. NeuroImage. 2008;41(2):636-47.

24. Bongers $\mathrm{P}$, et al. Being impulsive and obese makes susceptible to speeded detection of high calorie foods in the environment. Health Psychology (in press).

25. Frankort A et al. The craving stops before you feel it: neural correlates of chocolate craving during cue exposure with response prevention. Cereb Cortex. 2014;24(6):1589-600.

26. Nederkoorn C, Jansen A. Cue reactivity and regulation of food intake. Eat Behav. 2002;3(1):61-72.

27. Ferriday D, Brunstrom J. 'I just can't help myself': effects of foodcue exposure in overweight and lean individuals. Int $\mathrm{J}$ Obes. 2010;35(1):142-9.

28. Epstein LH, Paluch R, Coleman KJ. Differences in salivation to repeated food cues in obese and nonobese women. Psychosom Med. 1996;58(2):160-4.

29. Stice E et al. Relation of obesity to consummatory and anticipatory food reward. Physiol Behav. 2009;97(5):551-60.

30. Stice E et al. Reward circuitry responsivity to food predicts future increases in body mass: moderating effects of DRD2 and DRD4. NeuroImage. 2010;50(4):1618-25.

31. Demos KE, Heatherton TF, Kelley WM. Individual differences in nucleus accumbens activity to food and sexual images predict weight gain and sexual behavior. J Neurosci. 2012;32(16):5549-52.

32. Werthmann $\mathrm{J}$ et al. Can (not) take my eyes off it: attention bias for food in overweight participants. Health Psychol. 2011;30(5):561.

33. Braet C, Crombez G. Cognitive interference due to food cues in childhood obesity. J Clin Child Adolesc Psychol. 2003;32:32-9.

34. Roefs A, Houben K, Werthmann J. Desire for food and the power of mind, in the psychology of desire. In: Hofmann W, Nordgren LF, editors (in press).

35. Pursey K et al. Neural responses to visual food cues according to weight status: a systematic review of functional magnetic resonance imaging studies. Eat Behav. 2014;1:7.

36. Jansen A et al. Overweight children overeat after exposure to food cues. Eat Behav. 2003;4(2):197-209.

37. Nederkoorn C, Smulders FTY, Jansen A. Cephalic phase responses, craving and food intake in normal subjects. Appetite. 2000;35(1): 45-55.

38. Chao A et al. Food cravings, food intake, and weight status in a community-based sample. Eat Behav. 2014;15(3):478-82. 
39. Boutelle $\mathrm{KN}$ et al. Two novel treatments to reduce overeating in overweight children: a randomized controlled trial. J Consult Clin Psychol. 2011;79(6):759-71.

40. Jansen A et al. Decreased salivation to food cues in formerly obese successful dieters. Psychother Psychosom. 2010;79(4):257-8. The study showed that formerly obese successful dieters showed less cue-elicited salivation compared with currently obese unsuccessful dieters. Although this is a small and cross-sectional study, it suggests a link between lower cue reactivity and more successful dieting.

41. McCaffery JM et al. Differential functional magnetic resonance imaging response to food pictures in successful weight-loss maintainers relative to normal-weight and obese controls. Am J Clin Nutr. 2009;90(4):928-34.

42. Murdaugh DL et al. fMRI reactivity to high-calorie food pictures predicts short- and long-term outcome in a weight-loss program. NeuroImage. 2012;59(3):2709-21. This is a prospective fMRI study examining how cue-elicited brain activation is related to weight loss success.

43. Phelan $\mathrm{S}$ et al. Cognitive interference from food cues in weight loss maintainers, normal weight, and obese individuals. Obesity. 2011;19(1):69-73.

44. Deckersbach $\mathrm{T}$ et al. Pilot randomized trial demonstrating reversal of obesity-related abnormalities in reward system responsivity to food cues with a behavioral intervention. Nutr Diabetes. 2014;4: e129.

45. Ochner $\mathrm{CN}$ et al. Selective reduction in neural responses to high calorie foods following gastric bypass surgery. Ann Surg. 2011;253(3):502.

46. Winstanley CA, Eagle DM, Robbins TW. Behavioral models of impulsivity in relation to ADHD: translation between clinical and preclinical studies. Clin Psychol Rev. 2006;26(4):379-95.

47. Patton JH, Stanford MS. Factor structure of the Barratt impulsiveness scale. J Clin Psychol. 1995;51(6):768-74.

48. Whiteside SP et al. Validation of the UPPS impulsive behaviour scale: a four-factor model of impulsivity. Eur J Personal. 2005;19(7):559-74.

49. Guerrieri R et al. The influence of trait and induced state impulsivity on food intake in normal-weight healthy women. Appetite. 2007;49(1):66-73.

50. Appelhans BM et al. Delay discounting and intake of ready-to-eat and away-from-home foods in overweight and obese women. Appetite. 2012;59(2):576-84

51. Jasinska AJ et al. Impulsivity and inhibitory control deficits are associated with unhealthy eating in young adults. Appetite. 2012;59(3):738-47.

52. Appelhans BM et al. Inhibiting food reward: delay discounting, food reward sensitivity, and palatable food intake in overweight and obese women. Obesity. 2011;19(11):2175-82. This article found that food cue reactivity and impulsivity interact in predicting obese women's food intake. We think this points to an important issue that may account for inconsistent findings of associations between cue reactivity and eating behavior.

53. Guerrieri R et al. Inducing impulsivity leads high and low restrained eaters into overeating, whereas current dieters stick to their diet. Appetite. 2009;53(1):93-100. In this study, an experimental impulsivity induction was used and effects on food intake were studied. We believe this gives unique insights into the role of impulsivity in eating behavior.

54. Nederkoorn $\mathrm{C}$ et al. Why obese children cannot resist food: the role of impulsivity. Eat Behav. 2006;7(4):315-22.

55. Nederkoorn $\mathrm{C}$ et al. Impulsivity predicts treatment outcome in obese children. Behav Res Ther. 2007;45(5):1071-5.

56. Houben K, Nederkoorn C, Jansen A. Eating on impulse: the relation between overweight and food-specific inhibitory control. Obesity. 2014;22(5):E6-8.
57. Guerrieri R, Nederkoorn C, Jansen A. The interaction between impulsivity and a varied food environment: its influence on food intake and overweight. Int J Obes (Lond). 2008;32(4):708-14.

58. Nederkoorn $\mathrm{C}$ et al. Impulsivity in obese women. Appetite. 2006;47(2):253-6.

59. Nederkoorn $\mathrm{C}$ et al. The interactive effect of hunger and impulsivity on food intake and purchase in a virtual supermarket. Int J Obes. 2009;33(8):905-12.

60. Batterink L, Yokum S, Stice E. Body mass correlates inversely with inhibitory control in response to food among adolescent girls. An fMRI study. NeuroImage. 2010;52:1696-703.

61. Nederkoorn $\mathrm{C}$ et al. Specificity of the failure to inhibit responses in overweight children. Appetite. 2012;59:409-13.

62. Mobbs $\mathrm{O}$ et al. Cognitive deficits in obese persons with and without binge eating disorder. Investigation using a mental flexibility task. Appetite. 2011;57:263-71.

63. Mobbs $\mathrm{O}$ et al. Obesity and the four facets of impulsivity. Patient Educ Couns. 2010;79(3):372-7.

64. Davis $\mathrm{C}$ et al. Immediate pleasures and future consequences. A neuropsychological study of binge eating and obesity. Appetite. 2010;54(1):208-13.

65. Weller RE et al. Obese women show greater delay discounting than healthy-weight women. Appetite. 2008;51(3):563-9.

66. Stoeckel LE et al. Greater impulsivity is associated with decreased brain activation in obese women during a delay discounting task. Brain Imaging Behav. 2013;7(2):116-28.

67. Smith $\mathrm{E}$ et al. A review of the association between obesity and cognitive function across the lifespan: implications for novel approaches to prevention and treatment. Obes Rev. 2011;12(9): $740-55$.

68. Riggs NR et al. Relationships between executive cognitive function and lifetime substance use and obesity-related behaviors in fourth grade youth. Child Neuropsychol. 2012;18(1):1-11.

69. Allan JL, Johnston M, Campbell N. Missed by an inch or a mile? Predicting the size of intention-behaviour gap from measures of executive control. Psychol Health. 2011;26(6):635-50.

70. Schlam TR et al. Preschoolers' delay of gratification predicts their body mass 30 years later. J Pediatr. 2013;162(1):90-3.

71. Kishinevsky FI et al. fMRI reactivity on a delay discounting task predicts weight gain in obese women. Appetite. 2012;58(2): 582-92.

72. Moeller FG, Dougherty DM. Impulsivity and substance abuse: what is the connection? Addict Disord Treat. 2002;1(1):3-10.

73. Moeller FG et al. The impact of impulsivity on cocaine use and retention in treatment. J Subst Abus Treat. 2001;21(4):193-8.

74. Pauli-Pott $U$ et al. Does inhibitory control capacity in overweight and obese children and adolescents predict success in a weightreduction program? Eur Child Adolesc Psychiatry. 2010;19(2): $135-41$.

75. Weygandt $\mathrm{M}$ et al. The role of neural impulse control mechanisms for dietary success in obesity. NeuroImage. 2013;83:669-78.

76. Rollins BY, Dearing KK, Epstein LH. Delay discounting moderates the effect of food reinforcement on energy intake among non-obese women. Appetite. 2010;55(3):420-5.

77. Tetley AC, Brunstrom JM, Griffiths PL. The role of sensitivity to reward and impulsivity in food-cue reactivity. Eat Behav. 2010;11(3):138-43.

78. Liu S et al. Relationship between attentional bias to cocaine-related stimuli and impulsivity in cocaine-dependent subjects. Am J Drug Alcohol Abuse. 2011;37(2):117-22.

79. Velázquez-Sánchez $\mathrm{C}$ et al. High trait impulsivity predicts food addiction-like behavior in the rat. Neuropsychopharmacology. 2014:39:2463-72.

80. van den Akker $\mathrm{K}$ et al. Impulsivity makes more susceptible to overeating after contextual appetitive conditioning. Appetite. 2013;70:73-80. 
81. Papachristou H et al. Dissection of appetitive conditioning. Does impulsivity play a role? Appetite. 2013;69:46-53.

82. Veling $\mathrm{H}$ et al. Targeting impulsive processes of eating behavior via the internet. Effects on body weight. Appetite. 2014;78:102-9.

83. Houben $\mathrm{K}$, Jansen A. Training inhibitory control. A recipe for resisting sweet temptations. Appetite. 2011;56(2):345-9.

84. Houben $\mathrm{K}$ et al. Resisting temptation: decreasing alcohol-related affect and drinking behavior by training response inhibition. Drug Alcohol Depend. 2011;116:132-6.

85. Houben K, Wiers RW, Jansen A. Getting a grip on drinking behavior: training working memory to reduce alcohol abuse. Psychol Sci. 2011;22(7):968-75.

86. Verbeken $\mathrm{S}$ et al. Executive function training with game elements for obese children: a novel treatment to enhance self-regulatory abilities for weight-control. Behav Res Ther. 2013;51(6):290-9.

87. Di Pellegrino G, Magarelli S, Mengarelli F. Food pleasantness affects visual selective attention. Q J Exp Psychol. 2011;64:560-71.

88. Kemps E et al. Attentional retraining can reduce chocolate consumption. J Exp Psychol Appl. 2014;20:94-102.

89. Kakoschke N, Kemps E, Tiggeman M. Attentional bias modification encourages healthy eating. Eat Behav. 2014;15:120-4.

90. Werthmann J et al. Attention bias for chocolate increases chocolate consumption - an attention bias modification study. J Behav Ther Exp Psychiatry. 2014;45:136-43.

91. Boutelle $\mathrm{KN}$ et al. A pilot study evaluating a one-session attention modification training to decrease overeating in obese children. Appetite. 2014;76:180-5. 